\title{
Persistent fenestration may be a marker for physiologic intolerance after Fontan completion
}

\author{
Yasuhiro Kotani, MD, PhD, ${ }^{\mathrm{a}, \mathrm{c}}$ Devin Chetan, HBA, ${ }^{\mathrm{a}, \mathrm{c}}$ Arezou Saedi, MD, ${ }^{\mathrm{a}, \mathrm{c}}$ Jiaquan Zhu, MD, PhD, ${ }^{\mathrm{a}, \mathrm{c}}$ \\ Lars Grosse-Wortmann, MD, ${ }^{\mathrm{b}, \mathrm{d}}$ John G. Coles, MD, ${ }^{\mathrm{a}, \mathrm{c}}$ Christopher A. Caldarone, MD, ${ }^{\mathrm{a}, \mathrm{c}}$ \\ Glen S. Van Arsdell, MD, ${ }^{\mathrm{a}, \mathrm{c}}$ and Osami Honjo, $\mathrm{MD}, \mathrm{PhD}^{\mathrm{a}, \mathrm{c}}$
}

Background: We sought to evaluate the medium-term implications of fenestration status.

Methods: Between 1994 and 2012, 326 patients received an extracardiac Fontan (hospital mortality $\mathrm{n}=6$,
$1.8 \%$ ). A fenestration was routinely created $(\mathrm{n}=306,94 \%)$ unless the was technical difficulty. Three hundred
patients discharged with an open fenestration were included. The primary end points were death and Fontan
failure. Secondary outcomes were Fontan complications such as venovenous collaterals, protein-losing
enteropathy, pacemaker requirement, and arrhythmias.

Results: The fenestration was closed in 260 patients: 185 as a catheter intervention $(62 \%)$ and $75(25 \%)$ spontaneously. Forty patients $(13 \%)$ had the fenestration open at a median follow-up period of 5.05 years. Of these patients, catheter-based closure failed in $10(3 \%)$. There was no statistically significant difference in pre-Fontan hemodynamic parameters, such as pulmonary artery pressure and pulmonary vascular resistance between the patients with open fenestration and the ones with closed fenestration. Patients with an open fenestration had significantly more late deaths $(P<.001)$, Fontan failure $(P=.021)$, and Fontan complications $(P=.011)$ compared with those with a closed fenestration. Multivariable Cox regression revealed open fenestration $(P<.001)$ and indeterminate ventricular morphology $(P=.002)$ as risk factors for death/Fontan failure, and ventricular dysfunction $(P=.014)$ and open fenestration $(P=.009)$ as risk factors for Fontan complications.

Conclusions: Persistent fenestration was a marker for physiologic intolerance as noted by increased rates of mortality and a higher incidence of Fontan failure/complications. The specificity of pre-Fontan physiologic data for fenestration status may not have the fidelity needed for long-term care and thus, the consequences of decision making regarding fenestration status may not be determined until well after the operation. ( $\mathrm{J}$ Thorac Cardiovasc Surg 2014;148:2532-8)

See related commentary on pages 2538-9.

Surgical creation of a temporary communication between the Fontan pathway and the common atrium, ie, fenestration, was first introduced in the early 1990s as a strategy for highrisk Fontan candidates who had an estimated hospital survival of less than $85 \% .^{1}$ The rationale for fenestration was to lower the pressure in the Fontan circuit and increase cardiac output, thereby decreasing operative mortality and morbidity. ${ }^{1}$ Currently, a strategy of fenestration creation is

From the Divisions of Cardiovascular Surgery ${ }^{\mathrm{a}}$ and Cardiology, ${ }^{\mathrm{b}}$ The Labatt Family Heart Centre, The Hospital for Sick Children Toronto, Ontario, Canada; and the Departments of Surgery ${ }^{\mathrm{c}}$ and Paediatrics, ${ }^{\mathrm{d}}$ University of Toronto, Toronto, Ontario, Canada.

Disclosures: Authors have nothing to disclose with regard to commercial support.

Read at the 94th Annual Meeting of The American Association for Thoracic Surgery, Toronto, Ontario, Canada, April 26-30, 2014.

Received for publication April 28, 2014; revisions received May 31, 2014; accepted for publication June 27, 2014; available ahead of print Aug 16, 2014.

Address for reprints: Osami Honjo, MD, PhD, Division of Cardiovascular Surgery, The Hospital for Sick Children, 555 University Ave, Toronto, Ontario, Canada M5G 1X8 (E-mail: osami.honjo@sickkids.ca).

$0022-5223 / \$ 36.00$

Copyright $\odot 2014$ by The American Association for Thoracic Surgery

http://dx.doi.org/10.1016/j.jtcvs.2014.06.062 used worldwide as part of standard Fontan completion. ${ }^{2,3}$ Hospital survival for the Fontan operation in the current era is excellent after refinements in surgical strategy including fenestration and improvements in perioperative management.

The impact of fenestration on early clinical outcomes in standard-risk Fontan candidates has been studied in the last 2 decades. There was no survival benefit of fenestration reported and the survival of both fenestrated and nonfenestrated patients with standard-risk profiles was excellent. There were some positive effects of fenestration on secondary outcomes, including chest tube drainage, arrhythmias, and hospital stay, ${ }^{4-6}$ although some other retrospective studies showed no clinical benefit of fenestration. ${ }^{7,8}$ Much less evidence exists regarding the medium- to long-term effects of persistent fenestration on clinical outcomes. A retrospective study from the Pediatric Heart Network showed no additional benefit of fenestration on functional status and ventricular function at a mean follow-up of 7 to 8 years. ${ }^{7}$ A recent cardiac magnetic resonance study from our group showed that not only all inferior vena cava flow but also part of the superior vena cava flow is diverted through the fenestration in two thirds of the study population at a mean follow-up of 12 months, 


\section{Abbreviations and Acronyms \\ $\mathrm{AVV}=$ arterioventricular valve \\ NYHA $=$ New York Heart Association}

raising the possibility of potential adverse effects of persistent fenestration in the medium-term. ${ }^{9}$

Like many North American institutes, ${ }^{10}$ Fontan completion with an extracardiac tube graft with fenestration of 3 to $4 \mathrm{~mm}$ has been our consistent approach over the past 2 decades. Our intention has been to close the fenestration at 6 to 12 months after Fontan completion; nonetheless, several patients did not tolerate fenestration closure. We hypothesized that persistent fenestration does not have medium-term hemodynamic benefit but failure to close the fenestration may be an indicator of subsequent poor clinical outcomes. Thus, we investigated the impact of persistent fenestration on medium-term mortality and morbidity.

\section{METHODS}

A total of 326 patients who underwent extracardiac total cavopulmonary connection for single ventricle palliation at the Hospital for Sick Children between July 1994 and April 2012 were reviewed. Research Ethics Board approval was obtained. Patients who underwent a 1-stage Fontan operation or aortopulmonary connection Fontan operation were excluded. A fenestration was routinely created at our institution unless there was technical difficulty. A total of $306(94 \%)$ patients had a fenestration. Three hundred patients who had an open fenestration at the time of discharge were included in the study.

\section{Operative Technique and Fenestration Closure}

The surgical techniques for the Fontan operation have been described elsewhere. ${ }^{11}$ Briefly, standard normothermic or mild hypothermic cardiopulmonary bypass was used. Extracardiac total cavopulmonary connection was typically performed in an on-pump beating state without cardioplegic cardiac arrest unless other intracardiac procedures were required. A transverse incision was made on the inferior aspect of the central branch pulmonary artery and a tube graft was anastomosed. The inferior end of the graft was then anastomosed to the inferior vena cava. A fenestration incision was made on the tube graft as well as on the right atrial lateral wall, and a fenestration was created between the Fontan graft and the atrium by means of a direct side-by-side anastomosis without a bridging graft. A polytetrafluoroethylene tube ( $n=254)$, aortic homograft (in the 1990s; $n=67$ ), or another conduit $(\mathrm{n}=5)$ was used as the external conduit. The average conduit size was $20 \pm 1 \mathrm{~mm}$ (median, $21 \mathrm{~mm}$; range, 20-22 mm). The median size of the fenestration was $4 \mathrm{~mm}$ (range, $3.5-5 \mathrm{~mm}$ ).

Catheter-based fenestration closure was performed electively at 6 to 12 months if physiologically tolerated. After initial assessment at the outpatient clinic, patients were taken to the cardiac catheterizationlaboratory. Suitability for fenestration closure was decided based on changes in the central venous pressure (Fontan pressure), systemic blood pressure, and arterial oxygen saturation with test occlusion of the fenestration. Fenestration closure was performed with an Amplatzer septal occluder (AGA Medical, Golden Valley, Minn).

\section{Outcome Assessment}

All patient charts were independently reviewed by 3 investigators (YK, AS, and JZ). Echocardiography was used to assess whether or not the fenestration was open at latest follow-up and patients were divided into 2 groups: closed fenestration and open fenestration. Outcomes were compared between the 2 groups. Primary end points were death and Fontan failure, defined as Fontan takedown/revision, need for mechanical circulatory support, or transplantation. ${ }^{12-14}$ Secondary outcomes were Fontan complications, including venovenous collaterals, protein-losing enteropathy, pacemaker requirement, arteriovenous malformation, and arrhythmias. Echocardiographic images were reviewed and qualitative assessments of ventricular function and degree of arterioventricular valve (AVV) regurgitation were performed using a method described previously. ${ }^{15}$ Ventricular function was graded as normal, mildly reduced, moderately reduced, or severely reduced. The degree of AVV regurgitation was graded as none/trivial, mild, moderate, or severe.

\section{Statistical Analysis}

Continuous data are presented as median (interquartile range). Discrete data are presented as frequency (percentage). Differences between the groups were analyzed with the Mann-Whitney U test. Event frequencies were compared with the $\chi^{2}$ or Fisher test. Freedom from death/Fontan failure and Fontan complications were analyzed by Kaplan-Meier analysis and the logrank test. Univariable predictors for death/Fontan failure and Fontan complications were analyzed with a Cox regression model. Variables that were significant at the $P \leq .05$ level in univariable analysis were included in a multivariable Cox regression model. Statistical analysis was performed using SPSS version 17.0 statistical software (IBM Corporation, Armonk, NY).

\section{RESULTS}

Seventy-five $(25 \%)$ patients were identified as having a fenestration that closed spontaneously. A total of 195 $(65 \%)$ patients were brought to the catheterization laboratory for assessment of fenestration closure. Thirty $(10 \%)$ patients were considered too high risk for fenestration closure and were not assessed by test occlusion. Ten $(3 \%)$ of 195 patients failed balloon test occlusion because of high pulmonary artery pressure $(n=7)$ or hemodynamic instability $(\mathrm{n}=3)$. As a result, at a followup period of 5.05 (2.37-10.26) years, the fenestration was closed in 260 patients: 75 (25\%) by spontaneous closure and $185(62 \%)$ by catheter-based closure. Forty patients $(13 \%)$ had an open fenestration. There was no statistical difference between those with open and closed fenestration with respect to pre-Fontan status, including diagnosis, dominant ventricle, ventricular and AVV function, pulmonary artery pressure, and pulmonary vascular resistance (Table 1).

\section{Death and Fontan Failure}

Nine (3\%) patients died after discharge. Cause of death included circulatory failure in 2 patients, respiratory failure in 1 patient, multiorgan failure in 1 patient, thromboembolism in 1 patient, and unknown in 4 patients. Freedom from death in patients with a closed fenestration was significantly higher than that in patients with an open fenestration $(99.2 \%$ vs $74.5 \%$ at 5 years, $P<.001$; Figure $1, A)$. A comparison of 3 groups (catheter-based closure, $\mathrm{n}=185$; spontaneous closure, $\mathrm{n}=75$; and open, $\mathrm{n}=40$ ) showed that the mechanism of fenestration closure was not important but the presence of an open fenestration was associated with the lowest survival (spontaneous closure, 
TABLE 1. Preoperative profile

\begin{tabular}{|c|c|c|c|}
\hline & Closed fenestration $(n=260)$ & Open fenestration $(n=40)$ & $\boldsymbol{P}$ \\
\hline \multicolumn{4}{|l|}{ Patient demographics } \\
\hline Age, mo (range) & $37.7(31.2-45.5)$ & $40.8(33.8-54.5)$ & .071 \\
\hline Body weight, kg (range) & $13.7(12.4-15.3)$ & $14.2(12.8-16.2)$ & .191 \\
\hline Body surface area, $\mathrm{m}^{2}$ (range) & $0.55(0.51-0.59)$ & $0.56(0.52-0.61)$ & .326 \\
\hline Male/female gender, $\mathrm{n}(\%)$ & $166(64) / 94(36)$ & $25(63) / 15(37)$ & .869 \\
\hline \multicolumn{4}{|l|}{ Diagnosis, n (\%) } \\
\hline Hypoplastic left heart syndrome & $60(23)$ & $8(20)$ & .665 \\
\hline Double inlet left ventricle & $42(16)$ & $6(15)$ & .853 \\
\hline Tricuspid atresia & $42(16)$ & $6(15)$ & .853 \\
\hline Unbalanced atrioventricular septal defect & $6(2)$ & $3(8)$ & .104 \\
\hline Double outlet right ventricle & $15(6)$ & $5(13)$ & .162 \\
\hline Univentricular heart & $54(21)$ & $3(8)$ & .051 \\
\hline Isomerism & $23(9)$ & $6(15)$ & .220 \\
\hline Pulmonary artesia/intact ventricular septum & $18(7)$ & $3(8)$ & .749 \\
\hline \multicolumn{4}{|l|}{ Dominant ventricle, $\mathrm{n}(\%)$} \\
\hline Left & $154(59)$ & $19(48)$ & .162 \\
\hline Right & $91(35)$ & $19(48)$ & .127 \\
\hline Indeterminate & $15(6)$ & $2(5)$ & 1.000 \\
\hline \multicolumn{4}{|l|}{ Echocardiography } \\
\hline \multicolumn{4}{|l|}{ Atrioventricular valve regurgitation, $\mathrm{n}(\%)$} \\
\hline None/mild & $73(28) / 156(60)$ & $8(20) / 28(70)$ & $.278 / .237$ \\
\hline Moderate/severe & $30(12) / 0(0)$ & $3(8) / 0(0)$ & $.592 /-$ \\
\hline \multicolumn{4}{|l|}{ Ventricular function, $\mathrm{n}(\%)$} \\
\hline Normal/mildly decreased & $247(95) / 12(5)$ & $37(93) / 3(8)$ & $.433 / .433$ \\
\hline Moderately decreased/severely decreased & $0(0) / 0(0)$ & $0(0) / 0(0)$ & $-1-$ \\
\hline \multicolumn{4}{|l|}{ Catheterization } \\
\hline Mean pulmonary artery pressure, $\mathrm{mm} \mathrm{Hg}$ (range) & $10(9-12)$ & $11(9-12)$ & .489 \\
\hline Pulmonary vascular resistance, Wood units (range) & $1.8(1.0-2.5)$ & $1.9(1.6-2.4)$ & .491 \\
\hline
\end{tabular}

98.5\%; catheter-based closure, $94.5 \%$; and open fenestration; $74.5 \%$ at 5 years, $P<.001$; Figure $1, B)$. Three $(1 \%)$ patients had Fontan failure (1 patient with open fenestration and 2 patients with closed fenestration). Freedom from death/Fontan failure in patients with closed fenestration

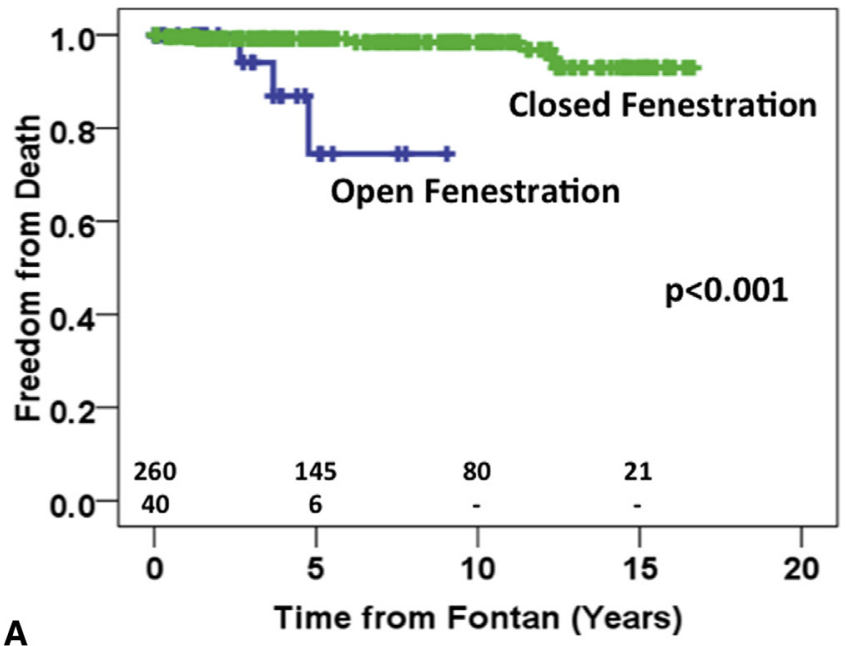

was significantly higher compared with patients with open fenestration $(99.2 \%$ vs $68.6 \%$ at 5 years, $P<.001)$.

Multivariable Cox regression revealed open fenestration $(P<.001)$ and indeterminate ventricular morphology $(P=.002)$ as risk factors for death/Fontan failure.

FIGURE 1. Freedom from death between open and closed fenestration (A) and stratified by mechanism of fenestration closure (B). The Kaplan-Meier curve showed that the mechanism of fenestration closure was not important but the presence of an open fenestration was associated with the lowest survival. 
TABLE 2. Fontan complications

\begin{tabular}{lccc}
\hline & $\begin{array}{c}\text { Closed } \\
\text { fenestration } \\
(\mathbf{n}=\mathbf{2 6 0}), \\
\mathbf{n}(\%)\end{array}$ & $\begin{array}{c}\text { Open } \\
\text { fenestration } \\
(\mathbf{n}=\mathbf{4 0}),\end{array}$ & \\
& $\mathbf{n}(\%)$ & $\boldsymbol{P}$ \\
\hline Ventricular dysfunction & $9(3)$ & $2(5)$ & .646 \\
Pulmonary vascular dysfunction & $2(1)$ & $4(10)$ & .003 \\
Mechanical problem & $21(8)$ & $1(3)$ & .330 \\
Thromboembolism & $8(3)$ & $2(5)$ & .628 \\
Arrhythmia & $6(2)$ & $1(3)$ & 1.000 \\
Others & & & \\
$\quad$ Protein-losing enteropathy & $4(2)$ & $1(3)$ & .514 \\
Plastic bronchitis & $0(0)$ & $1(3)$ & .133 \\
Arteriovenous malformation & $4(2)$ & $0(0)$ & 1.000 \\
Venovenous collaterals & $38(15)$ & $2(5)$ & .132 \\
Aortopulmonary collaterals & $13(5)$ & $1(2)$ & .702 \\
Arteriovenous fistula & $2(1)$ & $0(0)$ & 1.000 \\
$\quad$ Unknown & $3(1)$ & $0(0)$ & 1.000 \\
\hline
\end{tabular}

Pulmonary vascular dysfunction was defined as pulmonary artery pressure $>15 \mathrm{~mm} \mathrm{Hg}$ or pulmonary vascular resistance $>4$ Wood units.

\section{Fontan Complications}

One hundred twenty $(40 \%)$ patients developed Fontan complications. The occurrence of ventricular dysfunction, arrhythmias, and protein-losing enteropathy was comparable between patients with open and closed fenestration; however, pulmonary vascular dysfunction was more frequently seen in the open fenestration group $(10 \%$ vs $1 \%, P=.003$; Table 2). Freedom from Fontan complications in patients with a closed fenestration was significantly higher than that in patients with an open fenestration $(57.7 \%$ vs $13.9 \%$ at 5 years, $P=.011$; Figure $2, A)$. A comparison of 3 groups (catheter-based closure, spontaneous closure, and open fenestration) showed that the mechanism of fenestration closure was not important but the presence of an open fenestration was associated with the occurrence of Fontan complications $(P=.008$; Figure 2, B). Multivariable Cox regression showed ventricular dysfunction $(P=.014)$ and open fenestration $(P=.009)$ as risk factors for Fontan complications.

\section{Clinical Status}

A summary of the latest clinical status is shown in Table 3. The need for surgical or catheter reintervention was comparable between the groups (surgery, closed $7 \%$ vs open $11 \%, P=.498$; catheter, closed $36 \%$ vs open $24 \%, P=.175)$. Most $(84 \%)$ of the patients with a closed fenestration were New York Heart Association (NYHA) class I, whereas approximately half of the patients with an open fenestration were NYHA class I. Three $(8 \%)$ patients with an open fenestration were NYHA class IV. There was no difference in the rate of significant (moderate or more) AVV regurgitation and ventricular dysfunction between the groups. Patients with an open fenestration had higher pulmonary artery pressure and pulmonary vascular resistance (mean pulmonary artery pressure, 13.5 vs $12.0 \mathrm{~mm} \mathrm{Hg}, P=.004$; pulmonary vascular resistance, 4.5 vs 1.6 Wood units) at follow-up cardiac catheterization. Patients with open fenestration required more medication, especially anticoagulation.

\section{DISCUSSION}

The fenestrated Fontan strategy was introduced for high-risk patients with high pulmonary artery pressure and ventricular dysfunction. ${ }^{1,16}$ The advantages of fenestration include reduction of early Fontan failure and chest tube drainage and improvement in postoperative recovery. ${ }^{1,15,16}$ Recent refinements in patient selection,
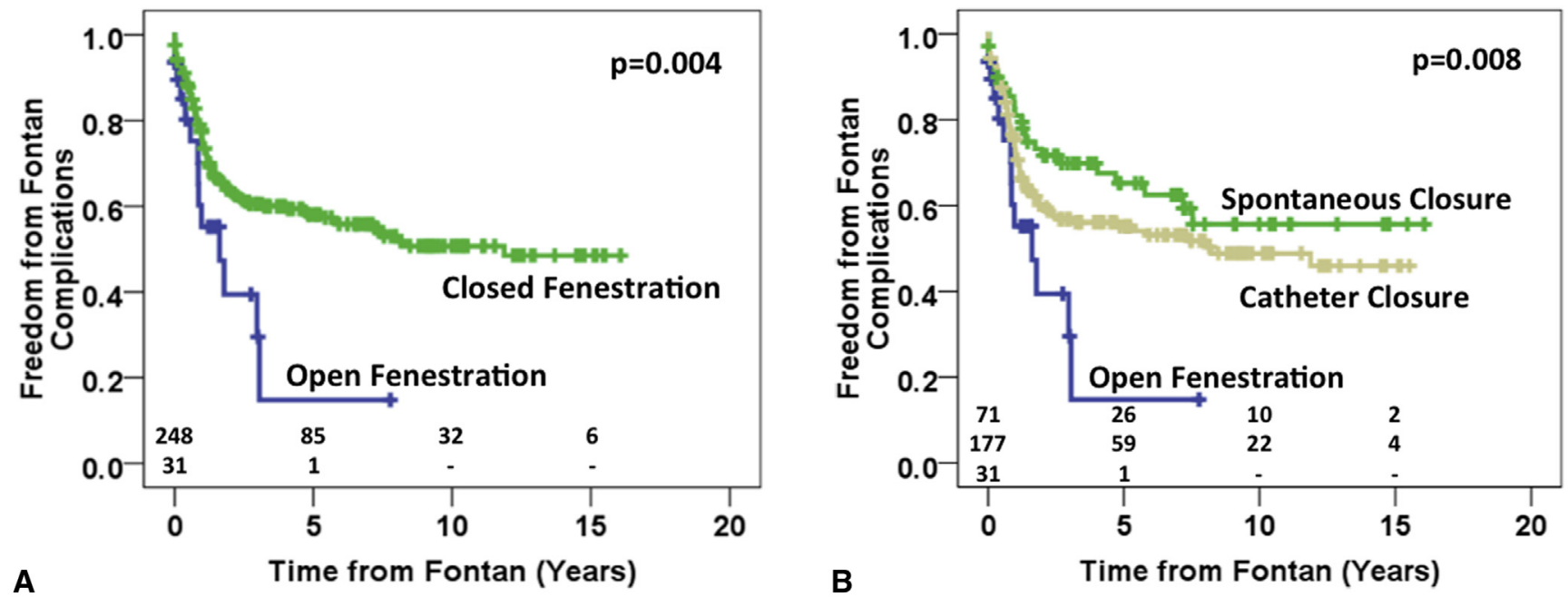

FIGURE 2. Freedom from Fontan complications between open and closed fenestration (A) and stratified by mechanism of fenestration closure (B). The Kaplan-Meier curve showed that the mechanism of fenestration closure was not important but the presence of an open fenestration was associated with the most complications. 
TABLE 3. Follow-up status

\begin{tabular}{|c|c|c|c|}
\hline & Closed fenestration $(n=260)$ & Open fenestration $(n=40)$ & $\boldsymbol{P}$ \\
\hline \multicolumn{4}{|l|}{ Reintervention, n (\%) } \\
\hline Surgery & $19(7)$ & $4(11)$ & .498 \\
\hline Catheter & $93(36)$ & $8(24)$ & .175 \\
\hline \multicolumn{4}{|l|}{ Follow-up data, n (\%) } \\
\hline \multicolumn{4}{|l|}{ NYHA class } \\
\hline $\mathrm{I} / \mathrm{II}$ & $218(84) / 18(7)$ & $22(55) / 6(15)$ & $<.001 / .043$ \\
\hline III/IV & $10(4) / 1(0)$ & $3(8) / 3(8)$ & $.198 / .006$ \\
\hline \multicolumn{4}{|l|}{ Echocardiography } \\
\hline \multicolumn{4}{|l|}{ Atrioventricular valve regurgitation, $\mathrm{n}(\%)$} \\
\hline None/mild & $53(20) / 168(65)$ & $8(20) / 27(68)$ & $.955 / .859$ \\
\hline Moderate/severe & $38(15) / 1(0)$ & $4(10) / 1(3)$ & $.624 / .249$ \\
\hline \multicolumn{4}{|l|}{ Ventricular function, $\mathrm{n}(\%)$} \\
\hline Normal/mildly reduced & $248(95) / 8(3)$ & $36(90) / 2(5)$ & $.245 / .628$ \\
\hline Moderately reduced/severely reduced & $3(1) / 1(0)$ & $0(0) / 2(5)$ & $1.000 / .048$ \\
\hline \multicolumn{4}{|l|}{ Catheterization at follow-up $(\mathrm{n}=231)$} \\
\hline Time from Fontan, y (range) & $1.11(0.72-1.77)$ & $1.09(0.86-2.21)$ & .914 \\
\hline Pulmonary vascular resistance, Wood units (range) & $1.6(1.1-2.5)$ & $4.5(3.8-4.6)$ & .001 \\
\hline Mean pulmonary artery pressure, $\mathrm{mm} \mathrm{Hg}$ (range) & $12.0(11.0-13.0)$ & $13.5(12.5-16.5)$ & .004 \\
\hline \multicolumn{4}{|l|}{ Heart transplantation, $\mathrm{n}(\%)$} \\
\hline Listed/transplanted & $16(6) / 3(1)$ & $4(10) / 2(5)$ & $.320 / .134$ \\
\hline Medication & $(\mathrm{n}=248)$ & $(\mathrm{n}=20)$ & \\
\hline \multicolumn{4}{|l|}{ Anticoagulation, n (\%) } \\
\hline None & $94(38)$ & $0(0)$ & $<.001$ \\
\hline Acetylsalicylic acid & $132(53)$ & $8(40)$ & .183 \\
\hline Warfarin & $19(8)$ & $11(55)$ & $<.001$ \\
\hline Low-molecular-weight heparin & $0(0)$ & $1(5)$ & .078 \\
\hline Clopidogrel & $3(1)$ & $0(0)$ & 1.000 \\
\hline Related to heart medication/none, $\mathrm{n}(\%)$ & $84(34) / 142(57)$ & $14(70) / 4(20)$ & $.001 / .002$ \\
\hline$\beta$-Blocker & $17(7)$ & $3(15)$ & .178 \\
\hline Digoxin & $14(6)$ & $1(5)$ & 1.000 \\
\hline Sildenafil & $2(1)$ & $5(25)$ & $<.001$ \\
\hline Acetylcholinesterase inhibitor & $69(28)$ & $9(45)$ & .104 \\
\hline Diuretics & $12(5)$ & $7(35)$ & $<.001$ \\
\hline Antiarrhythmics other than $\beta$-blocker & $0(0)$ & $1(5)$ & .075 \\
\hline Corticosteroids/nonrelated drugs & $6(2) / 52(21)$ & $2(10) / 8(40)$ & $.113 / .050$ \\
\hline
\end{tabular}

operative technique, and postoperative management brought a significant improvement in early outcome for even high-risk patients, raising the question whether fenestration is necessary for all patients including standard-risk patients. ${ }^{7,8}$ Because the medium- to long-term effects of fenestration are not well known, there is no universal agreement on whether routine surgical creation of fenestration is warranted.

We specifically examined the association between fenestration status and medium-term clinical outcomes, including death, Fontan failure, and Fontan complications. With our current strategy to routinely create and close the fenestration for all patients, $25 \%$ of patients were identified as having a spontaneously closed fenestration. Approximately two thirds of patients had a successful catheterbased fenestration closure. Thirty $(10 \%)$ patients still had a physiologic need for fenestration, including 3\% of patients in whom attempts to close the fenestration failed. Another key finding from this study is that open fenestrations were associated with more death, Fontan failure, and Fontan complications. There were no predictors for death, Fontan failure, and Fontan complications identified from the preoperative variables, such as ventricular function or pulmonary artery pressure, suggesting difficulty in determining whether or not fenestration is needed before Fontan completion. This, combined with the fact that late poor outcomes are mainly associated with ventricular dysfunction and pulmonary vascular problems, suggests that fenestration is important for maintaining the Fontan circulation for a small proportion of patients with unfavorable Fontan physiology.

\section{Spontaneous Fenestration Closure}

This study revealed that approximately one quarter of patients had spontaneous fenestration closure. This subgroup 
has the highest medium-term survival as well as freedom from any Fontan complications. Based on the clinical data, this subgroup has the most efficient Fontan physiology so they do not need to use the fenestration. The lack of effective flow through the fenestration is likely the main mechanism of spontaneous closure. The recent Pediatric Heart Network study showed that $19 \%$ of patients had spontaneous closure at 8 years after a Fontan operation. ${ }^{7}$ Other studies showed spontaneous closure rates of $8 \%$ at 2.4 years after surgery ${ }^{17}$ and $29 \%$ within 3 months after surgery. ${ }^{18}$ The difference in frequency of spontaneous closure may be associated with the institutional strategy for fenestration closure and surgical technique. Some centers routinely bring patients to the catheterization laboratory for fenestration closure at various time points after surgery and others do not. Our surgical technique to create the fenestration is direct side-by-side anastomosis between the Fontan graft and the common atrial wall. This technique may have a slightly higher spontaneous closure rate compared with fenestration with a bridging graft because of the right atrial trabeculation. Our data indicate that spontaneous closure of fenestration is generally associated with a favorable medium-term outcome.

The rationale for considering fenestration closure is that the positive effects of fenestration in Fontan physiology are limited to the early recovery period. Also there are some potential disadvantages of a long-term open fenestration, including persistent low arterial oxygenation, paradoxic embolization, and deleterious effects on neurologic and ventricular function. ${ }^{7,19}$ However, certain studies have shown no difference in functional status, ventricular function, and occurrence of stroke and thrombosis. ${ }^{10,20,21}$

\section{Fenestration and Late Clinical Outcomes}

This study shows that patients with an open fenestration had an increased risk of death, Fontan failure, and Fontan complications. Imielski and colleagues ${ }^{17}$ reported that there was no difference in event-free survival including heart transplantation, arrhythmia, and protein-losing enteropathy between the open and closed fenestration groups. These investigators suggested early fenestration closure before Fontan complications occur. ${ }^{17}$ They showed a significant number $(76 \%$ of the entire cohort) of patients had an open fenestration at a follow-up period of 2.4 years even though they routinely assessed suitability for catheterbased fenestration closure at 1 to 3 years after surgery. One possible reason is that an adverse event occurred before fenestration closure and such patients might be considered too high-risk to close the fenestration. Our strategy has been the routine creation of a fenestration followed by routine assessment for fenestration closure within 1 year. Although the optimal timing of fenestration closure has not been well elucidated, we believe that patients without any evidence of ventricular dysfunction and pulmonary vascular problems should be able to have fenestration closure once they recover from surgery. Patients, who are not candidates for fenestration closure or had a failed fenestration closure at 6 to 12 months need the fenestration to maintain Fontan circulation. Therefore, it is not surprising that patients with open fenestration have an increased risk for late adverse events.

Our data clearly indicate that failure to close the fenestration at 6 to 12 months is an indicator of subsequent Fontan complications, Fontan failure, and death. Those patients should be carefully monitored and proactively treated for their complications. Early induction of pulmonary vasodilator therapy and early consideration of Fontan takedown or heart transplantation may be warranted.

\section{Is Fenestration Necessary in All Patients?}

Surgical creation of fenestration in standard-risk patients has been controversial and remains controversial after conducting this study. There is a reasonable body of evidence that fenestration expedites postoperative recovery by lowering Fontan pressure and increasing cardiac output in high-risk patients. ${ }^{1,4,20}$ The only prospective randomized study to assess the need for fenestration in standard-risk patients showed decreased chest tube drainage, less requirements for additional procedures, and subsequent shorter hospitalization. ${ }^{4}$ Some studies, however, showed no difference in these postoperative clinical outcomes in standard-risk patients, suggesting fenestration is not required in most patients. ${ }^{7,8}$ Thompson and colleagues ${ }^{7}$ create a fenestration based on a postbypass Fontan pressure guideline.

One important result from our study is that all pre-Fontan factors including diagnosis, ventricular function, pulmonary artery pressure, and pulmonary vascular resistance did not predict failure to close the fenestration. This result highlights the difficulty with predicting who is going to need a fenestration in the long-term and who is going to have subsequent Fontan failure. Our previous study also showed that pulmonary vascular resistance is not always a discriminator of subsequent successful fenestration closure. $^{9}$ A significant contribution to cardiac output with fenestration flow is important to distinguish patients who are eligible for fenestration closure. This is also difficult to know before Fontan completion. Given the unpredictable nature of fenestration requirements, routine creation of a fenestration in the standard-risk patient still seems to be a reasonable strategy, recognizing that many patients do not need fenestration at any point after Fontan completion and complications associated with fenestration closure are not negligible.

\section{Study Limitations}

The main limitation of this study is its retrospective nature. 


\section{CONCLUSIONS}

Spontaneous fenestration closure is likely an indicator of good Fontan physiology and a favorable clinical outcome. Persistent fenestration at 6 to 12 months was a marker for physiologic intolerance as noted by higher mortality and a higher incidence of Fontan failure/complications. Late fenestration patency was clearly important in $3 \%$ of the total population. The specificity of pre-Fontan physiologic data for fenestration status may not have the fidelity needed for long-term care and thus, the consequences of decision making regarding fenestration status may not be determined until well after the operation.

\section{References}

1. Bridges ND, Lock JE, Castaneda AR. Baffle fenestration with subsequent transcatheter closure. Modification of the Fontan operation for patients at increased risk. Circulation. 1990;82:1681-9.

2. Kouchoukos N. Cardiac Surgery. London: Churchill Livingstone; 2003.

3. Anderson R. Pediatric Cardiology. London: Churchill Livingstone; 2009.

4. Lemler MS, Scott WA, Leonard SR, Stromberg D, Ramaciotti C. Fenestration improves clinical outcome of the Fontan procedure: a prospective, randomized study. Circulation. 2002;105:207-12.

5. Gentle TL, Mayer JE, Gauvreau K, Newburger JW, Lock JE, Kupferschmid JP, et al. Fontan operation in five hundred consecutive patients: factors influencing early and late outcome. J Thorac Cardiovasc Surg. 1997;114:376-91.

6. Sinha P, Zurakowski D, He D, Yerebakan C, Freedenberg V, Moak JP, et al. Intra/extracardiac fenestrated modification leads to lower incidence of arrhythmias after the Fontan operation. J Thorac Cardiovasc Surg. 2013;145: 678-82.

7. Thompson LD, Petrossian E, McElhinney DB, Abrikosova NA, Moore P, Reddy VM, et al. Is it necessary to routinely fenestrate an extracardiac fontan? J Am Coll Cardiol. 1999;34:539-44.

8. Hsu DT, Quaegebeur JM, Ing FF, Selber EJ, Lamour JM, Gersony WM. Outcome after the single-stage, nonfenestrated Fontan procedure. Circulation. 1997;96: II-335-40.
9. Grosse-Wortmann L, Dragulescu A, Drolet C, Chaturvedi R, Kotani Y, Mertens L, et al. Determinants and clinical significance of flow via the fenestration in the Fontan pathway: a multimodality study. Int J Cardiol. 2013; 168:811-7.

10. Atz AM, Travison TG, McCrindle BW, Mahony L, Quartermain M, Williams RV, et al. Late status of Fontan patients with persistent surgical fenestration. J Am Coll Cardiol. 2011;57:2437-43.

11. Azakie A, McCrindle BW, Van Arsdell G, Benson LN, Coles J, Hamilton R, et al. Extracardiac conduit versus lateral tunnel cavopulmonary connections at a single institution: impact on outcomes. J Thorac Cardiovasc Surg. 2001;122: 1219-28.

12. Part IV-the dictionary of definitions of complications associated with the treatment of patients with congenital cardiac disease. Cardiol Young. 2008; 18(Suppl 2):282-530.

13. Stewart RD, Pasquali SK, Jacobs JP, Benjamin DK, Jaggers J, Cheng J, et al. Contemporary Fontan operation: association between early outcome and type of cardiopulmonary connection. Ann Thorac Surg. 2013;93:1254-61.

14. Wallace MC, Jaggers J, Li JS, Marshall L, Jacobs JP, Benjamin DK, et al. Center variation in patient age and weight at Fontan operation and impact on post-operative outcomes. Ann Thorac Surg. 2011;91:1445-52.

15. Kotani Y, Chetan D, Atlin CR, Mertens LL, Jegatheeswaran A, Caldarone CA, et al. Longevity and durability of atrioventricular valve repair in singleventricle patients. Ann Thorac Surg. 2012;94:2061-9.

16. Bridges ND, Mayer JE, Lock JE, Jonas RA, Hanley FL, Keane JF, et al. Effect of baffle fenestration on outcome of the modified Fontan operation. Circulation. 1992;86:1762-9.

17. Imielski BR, Woods RK, Mussatto KA, Cao Y, Simpson PM, Tweddell JS. Fontan fenestration closure and event-free survival. J Thorac Cardiovasc Surg. 2013;145:183-7.

18. Sommer RJ, Recto M, Golinko RJ, Griepp RB. Transcatheter coil occlusion of surgical fenestration after Fontan operation. Circulation. 1996;94:249-52.

19. Cetta F, Feldt RH, O'Leary PW, Mair DD, Warnes CA, Driscoll DJ, et al. Improved early morbidity and mortality after Fontan operation: the Mayo Clinic experience, 1987 to 1992. J Am Coll Cardiol. 1996;28:480-6.

20. Airan B, Sharma R, Choudhary SK, Mohanty SR, Bhan A, Chowdhari UK, et al. Univentricular repair: is routine fenestration justified? Ann Thorac Surg. 2000; 69:1900-6

21. Du Plessis AJ, Chang AC, Wessel DL, Lock JE, Wernovsky G, Newburger JW, et al. Cerebrovascular accidents following the Fontan operation. Pediatr Neurol. 1995;12:230-6.

\title{
EDITORIAL COMMENTARY
}

\section{To fenestrate or not: The Fontan debate gets more complicated}

\author{
Charles D. Fraser, Jr, MD
}

See related article on pages 2532-8.

From the Texas Children's Hospital and Baylor College of Medicine, Houston, Tex. Disclosures: Author has nothing to disclose with regard to commercial support. Received for publication Oct 4, 2014; accepted for publication Oct 6, 2014

Address for reprints: Charles D. Fraser, Jr, MD, Texas Children's Hospital, Office of the Surgeon-in-Chief, 6621 Fannin St, MC19345H, Houston, TX 77030 (E-mail: cdfraser@texaschildrens.org).

J Thorac Cardiovasc Surg 2014;148:2538-9

$0022-5223 / \$ 36.00$

Copyright (c) 2014 by The American Association for Thoracic Surgery

http://dx.doi.org/10.1016/j.jtcvs.2014.10.022
Expectations for acute postoperative outcomes after Fontan's palliation for single ventricle physiology have increased dramatically during the past 2 decades. Many centers report operative mortality rates of $1 \%$ or less with progressively shorter hospital lengths of stay and fewer perioperative complications. Patients are routinely extubated either in the operating room or within the first several postoperative hours and it is not uncommon for patients to stay in an intensive care unit for fewer than 24 hours. For those of us who grew up in the earlier era of Fontan procedures, particularly in the era of atriopulmonary connections, the differences in acute outcomes are nothing short of miraculous. 